\title{
Seroprevalence and risk factors of Trypanosoma evansi infection in horses in Peninsular Malaysia
}

\begin{abstract}
A cross-sectional study was designed to assess the seroprevalence and risk factors associated with Trypanosoma evansi infection among horses, using a total of 527 blood samples obtained from eight states in Peninsular Malaysia. A structured questionnaire was used to collect data on risk factors associated with $\mathrm{T}$. evansi seroprevalence. The overall seroprevalence detected by card agglutination test for $\mathrm{T}$. evansi (CATT/T. evansi) was $13.90 \%$ (73/527, CI: $11.2-17.1 \%)$. Female and exogenous horses showed a higher risk in association with the disease seroprevalence compared to other groups. The majority of the horse owners were not familiar with surra $(85.30 \%)$. However, most of them were very cautious with the health of their animals. In conclusion, this study showed that T. evansi occurred in low frequency among horses in Peninsular Malaysia, and the good management system adopted by horse owners was probably responsible for the low T. evansi occurrence.
\end{abstract}

Keyword: Trypanosoma evansi; Horses; Seroprevalence; Peninsular Malaysia 